\title{
Evaluation of the Susceptibility Profiles, Genetic Similarity and Presence of qnr Gene in Escherichia coli Resistant to Ciprofloxacin Isolated in Brazilian Hospitals
}

Andrea S. Pereira ${ }^{1}$, Soraya S. Andrade ${ }^{1}$, Jussimara Monteiro ${ }^{1}$, Helio S. Sader ${ }^{1,2}$, Antonio C.C. Pignatari ${ }^{1}$ and Ana C. Gales $^{1}$ Special Laboratory of Microbiology, Federal University of São Paulo, SP, Brazil; ${ }^{2}$ JMI Laboratories, North Liberty, IA, USA

Increasing quinolone resistance has been reported worldwide, mainly among clinical isolates of Escherichia coli. The objectives of this study were to determine the susceptibility profile, the genetic relatedness, and the prevalence of the qnr gene among ciprofloxacin-resistant Escherichia coli isolated from distinct Brazilian hospitals. A total of 144 ciprofloxacin-resistant Escherichia coli were isolated from 17 Brazilian hospitals between January/2002 and June/2003. The antimicrobial susceptibility testing was performed by microdilution according to NCCLS. The presence of the qnr gene was initially screened by colony blotting, and then confirmed by PCR followed by DNA sequencing. Ninety-five urinary ciprofloxacin-resistant Escherichia coli were further selected for molecular typing by pulsed-field gel electrophoresis (PFGE). Imipenem and meropenem showed the highest susceptibility rates (100.0\% for both compounds) followed by amikacin (91.0\%) and piperacillin/tazobactan (84.8\%). A single ciprofloxacinresistant Escherichia coli isolate was positive for qnr among the 144 ciprofloxacin-resistant Escherichia coli. Fortysix PFGE patterns were observed among the 95 ciprofloxacin-resistant Escherichia coli type. This study shows that therapeutic options are limited for treatment of ciprofloxacin-resistant Escherichia coli due to the presence of additional mechanisms of antimicrobial resistance, such as ESBL production. The qnr gene was uncommon among ciprofloxacin-resistant Escherichia coli clinical isolates, but its identification might indicate the emergence of this mechanism of quinolone resistance in Brazil. The great genomic variability found among the ciprofloxacin-resistant Escherichia coli highlights the importance of the appropriate use of quinolone to restrict the selection of resistant isolates.

Key-Words: Quinolone resistance, Escherichia coli, PFGE, qnr.

Escherichia coli is a leading cause of nosocomial as well as community-acquired infections, especially urinary tract infections (UTI) [1,2]. Recent studies have shown a considerable increase in the prevalence of resistance to ciprofloxacin among clinical isolates of Escherichia coli $[3,4]$. Although quinolone resistance generally results from chromosomal mutation in gyrA and parC genes, recent studies showed that low-level resistance can be also plasmid mediated by the acquisition of the $q n r$ gene mediated by plasmid pMG252 [5-7]. This plasmid was first report in 1998 in a Klebsiella pneumoniae clinical strain isolated in 1994 at the University of Alabama at Birmingham Medical Center [8], but has still not been detected among isolates from South America.

The objectives of this study were to determine the susceptibility profile, the genetic relatedness, and the frequency of the qnr gene among ciprofloxacin-resistant Escherichia coli (CR-EC) isolated from distinct Brazilian hospitals.

\section{Materials and Methods \\ Participant Institutions \\ A total of seventeen medical centers participated in the study. The centers were located throughout eight Brazilians \\ Received on 16 August 2006; revised 15 December 2006. \\ Address for correspondence: Dr. Andrea S. Pereira. Laboratório Especial de Microbiologia Clínica - LEMC. Disciplina de Infectologia. Universidade Federal de São Pulo. Rua Leandro Dupret, 188, São Paulo, SP - Zip code: 04025-010. Phone/FAX: (11) 5571-5180/5081-2965. E-mail: andrea.pereira@lemc.com.br}

The Brazilian Journal of Infectious Diseases 2007;11(1):40-43. (C) 2007 by The Brazilian Journal of Infectious Diseases and Contexto Publishing. All rights reserved. estates: Bahia (one center), Minas Gerais (two centers), Brasília (one center), Ceará (two centers), Maranhão (one center), Paraná (two centers), Rio Grande do Sul (one center), Santa Catarina (one center) and São Paulo (six centers) (Figure 1).

\section{Bacterial Isolates}

The Brazilian hospitals were requested to send CR-EC isolates to the coordinating laboratory. From January 2002 to June 2003, a total of 144 clinical isolates of CR-EC were isolated from the 17 hospital-based laboratories. Only one isolate per patient was included in the study. The isolates were identified at the participating institution by the routine methodology in use at each laboratory. Confirmation of species identification was performed by biochemical conventional methods if necessary.

\section{Antimicrobial Susceptibility}

Antimicrobial susceptibility testing was performed using the reference broth microdilution method as described by the National Committee for Clinical Laboratory Standards (NCCLS 2003). The minimal inhibitory concentration (MIC) was defined as the lowest antimicrobial concentration able to totally inhibit bacterial growth. Quality control was performed by testing Escherichia coli ATCC 25922, Pseudomonas aeruginosa ATCC 27853 and Klebsiella pneumoniae ATCC 70603.

According to NCCLS criteria, MICs $\geq 2 \mu \mathrm{g} / \mathrm{mL}$ for cefotaxime and/or ceftriaxone and/or ceftazidime and/or aztreonam were considered as extended-spectrum $\beta$-lactamase (ESBL) producers [9]. The ESBL phenotype was confirmed by using the addition of clavulanic acid (Oxoid ${ }^{\circledR}$ Basingstoke, England) according to manufacture recommendation. 
Screening for the qnr Gene in Clinical Strains EC-QR

All clinical strains of EC-QR were screened for the $q n r$ gene by the colony blotting and Southern hybridization methods using the kit "Ready to go" (Amersham Pharmacia Biotech Inc., New Jersey, EUA), in accordance with the manufacture's recommendations. The $q n r$ probe was made from plasmid pMG252 by PCR amplification with primers sense 5' GATAAAGTTTTTCAGCAAGAGG 3' and anti-sense 5' ATCCAGATCGGCAAAGGTTA3'.

\section{PCR Detection of qnr Gene}

The gene was amplified by using primers QP1 (5' GATAAAGTTTTTCAGCAAGAGG 3'starting at the 12th qnr nucleotide) and QP2 (5’ATCCAGATCGGCAAA GGTTA3’) to produce a 593-bp product, as described by Jacoby et al. [6]. PCR conditions were $94^{\circ} \mathrm{C}$ for $1 \mathrm{~min}, 57^{\circ} \mathrm{C}$ for $30 \mathrm{~s}$, and $72^{\circ} \mathrm{C}$ for $1 \mathrm{~min}$ for 30 cycles.

\section{Quality Control}

The QnrA-producing Klebsiella pneumoniae pMG252 and Escherichia coli ATCC 25922 were used as positive and negative quality controls for hybridization and PCR experiments, respectively.

\section{Nucleotide Sequence of qnr Gene}

Sequencing was performed using Big Dye terminator version II (BigDye ${ }^{\mathrm{TM}}$ Terminators v2.0 - Cycle Sequencing Kit - Applied biosystems-ABI PRISM ${ }^{\circledR}$, California, EUA) and in equipment an ABI PRISM 377 DNAAnalyzer (Perkin Elmer ${ }^{\circledR}$, California, EUA) reading was performed. The nucleotide sequence has been submitted to the GenBank database and assigned accession number AM295981.

\section{Evaluation of Genetic Similarity in CR-EC Isolates}

CR-EC collected from urinary tract infections were selected for molecular typing by Pulsed-field gel electrophoresis (PFGE). From each medical center, a maximum of $10 \mathrm{CR}-\mathrm{EC}$ isolates were included for analysis. Genomic DNA was prepared for restriction fragments analysis as described by Pfaller [10] and digested with SpeI restriction enzyme (New England Biolab, Inc., Beverly, USA). PFGE was performed on the CHEF DR III (Bio-Rad, Richmond, CA), under the following conditions: $1 \%$ agarose, $13^{\circ} \mathrm{C}, 200 \mathrm{~V}$ for $23 \mathrm{~h}$. The obtained fragments were analyzed according to the Pfaller criteria (1992) [10].

\section{Results}

Among the 144 CR-EC, a total of $76.4 \%$ and 3.5\% were isolated, respectively, from patients with urinary tract infections and bloodstream infections. The remaining isolates $(n=29)$ were recovered from peritoneal fluids, bronchoalveolar wash, catheter tip and multiple abscesses. A total of $43 \%$ of all CR-EC from urinary tract infections were collected from outpatients, reflecting the increasing frequency of quinolone resistance among community-acquired infections in the
Brazilian medical centers. Although data from the SENTRY program in Latin America has already detected an increasing frequency of CR-EC in this region, these data were collected from hospitalized patients [1]. This high frequency of CR-EC found in the present study contrasts with data from other countries, such as the United States, where the prevalence of quinolone resistance among UTI isolates ranges from $0.7 \%$ in 1996 to $2.5 \%$ in 2001 [2]. The current data may be related to the increased use of quinolones for the treatment of communityacquired infections, such as UTI and respiratory tract infections in Brazil.

The antimicrobial activity and susceptibility profile of all 144 CR-EC is summarized in Table 1. The best coverage was achieved by the tested carbapenems, imipenem and meropenem (100.0\%), followed by amikacin (91\%) and ceftazidime (91\%), and piperacilin/tazobactan (84.8\%). The decreased susceptibility rates to cefoxitin (47.6\%) may reflect the presence of alternate resistance mechanisms, such as alterations in outer membrane permeability, AmpC hyperproduction $[11,12]$ or the acquisition of plasmid-borne genes mediating the expression of other class $C \beta$-lactamases [13-15].

Based on the NCCLS/CLSI criteria, 22.9\% $(n=33)$ of the CR-EC isolates collected between 2002 and 2003 could be considered ESBL producers. The production of ESBL was variable according to sex, age and infection origin, as shown in Table 2. Although there was no difference in relation to sex and infection origin, isolates recovered from patients older than 60 years old presented an increased chance of harboring the ESBL phenotype $(p=0,006)$. These data is in accordance to a previous study by Eom at al. (2002) [16], where advanced age was a risk factor associated with the acquisition of quinolone-resistant E. coli among patients with UTI.

In this study, CR-EC harboring the ESBL phenotype could be detected in most of participating medical centers in Brazil (76.4\%), indicating these multi-drug-resistant isolates are a reality in many geographical regions within the country. This increased ESBL production rate among CR-EC is worrisome, since the treatment of these multi-drug-resistant isolates is limited to the available carbapenems.

A total 144 CR-EC isolates were screened by colony blotting and Southern hybridization method. Only one clinical isolate yielded a hybridization signal with the qnr gene probe as showed in the Figure 2. This isolate was sent by a hospital in Porto Alegre, from an 80 year- old patient with urinary tract infection. Among the tested antimicrobial agents, this sample was resistant to ciprofloxacin, nalidixic acid, piperacillin/ tazobactam, and cefoxitin. The ESBL screening and confirmatory tests were both negative for this specific sample. The qnr gene was sought by PCR by using specific primers in this strain, which was positive for screening test (Figure 3). After performing the nucleotide sequencing, a sequence of 493 bp fragment was obtained, identical to those of the original plasmid pMG252, as previously described by Jacoby et al. in 2003 [6]. 
Table 1. Antimicrobial potency and spectrum for selected antimicrobial agents tested against CR-EC isolates in 17 Brazilian laboratory-based hospitals

\begin{tabular}{llll}
\hline Antimicrobial agents & $\mu \mathrm{g} / \mathrm{mL}$ & \% Susceptibility \\
\cline { 2 - 3 } & $\mathrm{MIC}_{50} \mathrm{MIC}_{90}$ \\
\hline
\end{tabular}

\section{$\beta$-lactams}

$\begin{array}{llll}\text { Aztreonam } & \leq 1 & >32 & 77.9 \\ \text { Ceftriaxone } & \leq 1 & >64 & 75.9 \\ \text { Ceftazidime } & \leq 1 & 8 & 91.0 \\ \text { Cefoxitin } & 4 & 32 & 52.4 \\ \text { Cefepime } & \leq 1 & >32 & 80.0 \\ \text { Ampicillin/Sulbactam } & 32 & >64 & 27.6 \\ \text { Piperacilin/Tazobactam } \leq 8 & 128 & 84.8\end{array}$

\section{Carbapenems}

$\begin{array}{llll}\text { Imipenem } & \leq 0,25 & \leq 0,25 & 100.0 \\ \text { Meropenem } & \leq 0,25 & \leq 0,25 & 100.0\end{array}$

\section{Quinolones}

$\begin{array}{llll}\text { Nalidix acid } & \geq 32 & >32 & 0.0 \\ \text { Gatifloxacin } & \geq 8 & \geq 8 & 3.4\end{array}$

\section{Aminoglycosides}

Gentamicin $\quad \leq 2 \quad>16 \quad 65.7$

$\begin{array}{llll}\text { Amikacin } & \leq 8 & 16 & 91.0\end{array}$

Figure 1. The participating medical centers are marked on the map with a circle signal.

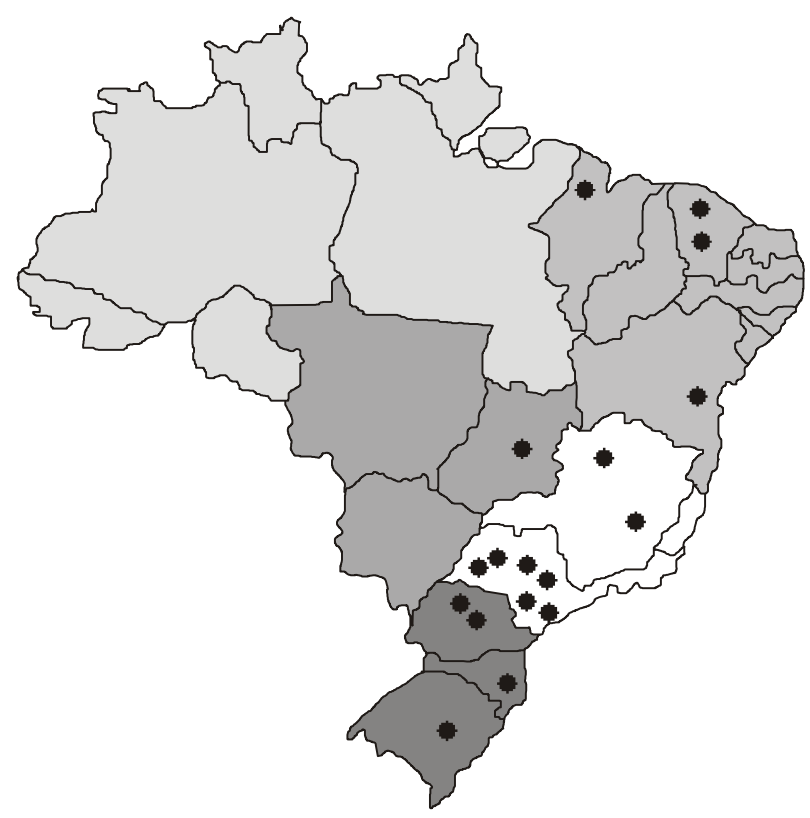

Table 2. Demographic characteristics of the 33 ciprofloxacinresistant ESBL-producing E. coli isolates

\begin{tabular}{lll}
\hline \multicolumn{3}{c}{ ESBL producing (\%) } \\
\hline Sex & Male & $16(48.5)$ \\
& Female & $17(51.5)$ \\
Age $^{\text {a }}$ & & \\
& $\leq 1$ & $2(6.1)$ \\
& $2-12$ & $1(3.0)$ \\
& $13-60$ & $8(24.2)$ \\
& $>60$ & $16(48.5)$ \\
Infection origin & \\
\multicolumn{2}{l}{ Community } & $5(15.2)$ \\
\multicolumn{2}{l}{ Nosocomial } & $7(21.2)$ \\
\multicolumn{2}{l}{ Indeterminated } & $21(63.6)$ \\
\hline a Age information was missing from 6 isolates.
\end{tabular}

Figure 2. Screening for the qnr gene by colony blotting technique.

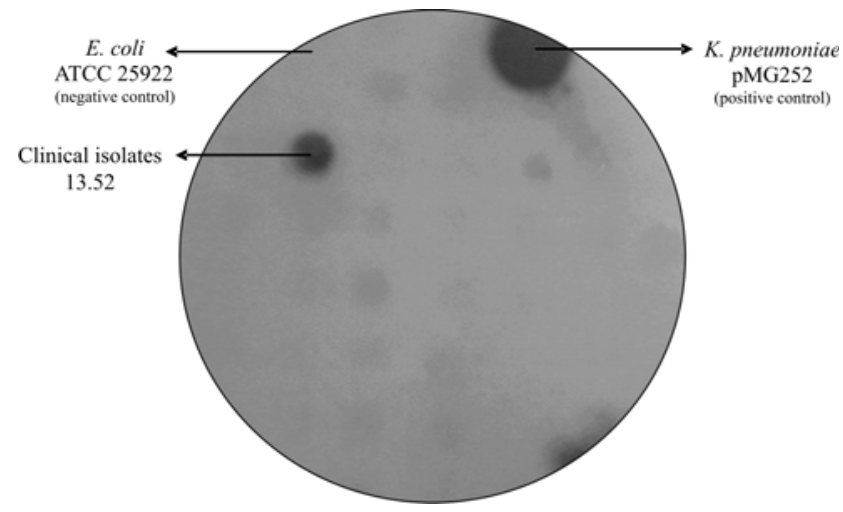

Figure 3. Detection of qnr gene by PCR. Line 1, Molecular weight marker; Line 2, negative quality control strain (ATCC 25922 E. coli); Line 3, clinical isolate (13.52); Line 4, positive quality control strain (pMG252 K. pneumoniae) and Line 5, master-mix solution.

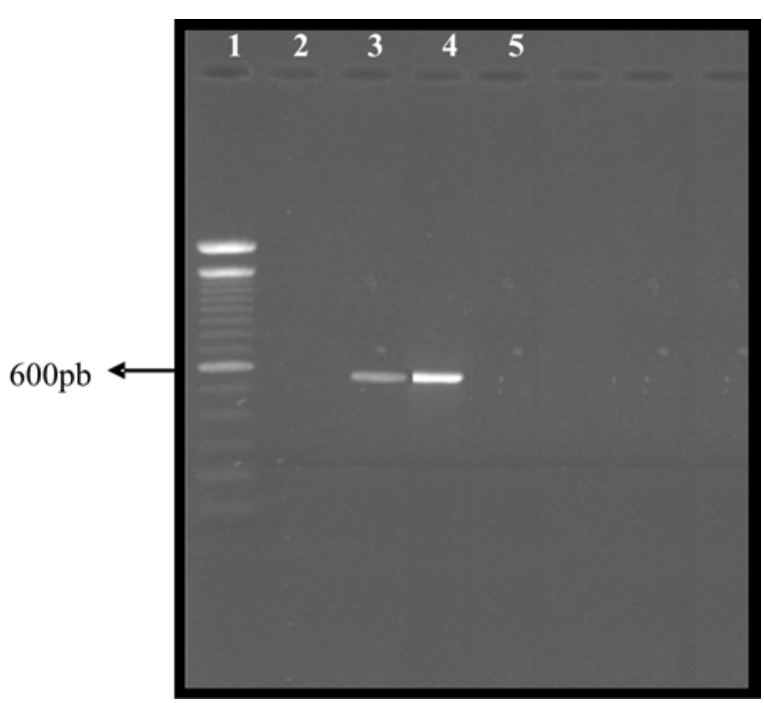




\section{Discussion}

The current study identified, for the first time, the presence of the qnr gene in a clinical isolate of $E$. coli from a city in Brazil. This finding may reflect the ability and the possibility of acquisition of this unusual genotype by Brazilian isolates of $E$. coli. The low frequency of qnr gene isolation was also described by other authors. After evaluating a total of $91 \mathrm{E}$. coli isolates from the United States, Jacoby et al. (2003) [6] could identify only one isolate harboring the qnr gene. In China, a study by Wang et al. [7] also found a low prevalence (8\%) of this genotype among the high-level quinolone resistant E. coli.

Similar PFGE profiles were detected among patients from the same medical center, possibly indicating a patient-topatient transmission of resistant isolates. On the other hand, a total of 46 distinct PFGE patterns were detected among the 95 CR-EC isolated from urinary tract infections, indicating an elevated genetic diversity. Therefore, this increased frequency of CR-EC may be associated with the selection of resistant $E$. coli isolates from the patients' own flora. These isolates may be associated to the previous use of fluoroquinolones by the patient, or by the ingestion of food products contaminated with resistant pathogens. This last hypothesis was confirmed by a study in Spain, which found an elevated number of quinolone-resistant E. coli colonizing poultry and pork [3]. Additional PFGE studies are necessary to establish the temporal and geographical distribution of these resistant isolates, in order to determine the mode of dissemination.

Fluoroquinolone usage and prescription should be restricted among community and hospitalized patients, mainly among populations with an increased risk of developing this resistance phenotype. Additional surveillance studies are necessary to understand the dissemination of quinolone resistance in Brazil, to help implementing appropriate control measures, as well as to guide the adequate use of antimicrobial agents in this geographic region.

\section{Acknowledgements}

We would like thank Dr. Afonso Barth and Lalitagauri Deshpande for providing the clinical isolates and positive quality control strain, respectively. This study was supported by the Conselho Nacional de Desenvolvimento Científico e Tecnológico-CNPq.

\section{References}

1. Gales A.C. Urinary tract infection trends in Latin American hospitals: report from the SENTRY antimicrobial surveillance program (1997-2000). Diagnostic Microbiolology and Infectious Disease 2002;44:273-80.

2. Karlowsky J.A., Kelly L.J., Thornsberry C., et al. Trends in antimicrobial resistance among urinary tract infection isolates of Escherichia coli from female outpatients in the United States. Antimicrobial Agents and Chemotherapy 2002;46:2540-5.

3. Garau J., Xercavins M., Rodríguez-Carballeira M., et al. Emergence and dissemination of quinolone-resistant Escherichia coli in the community. Antimicrobial Agents and Chemotherapy 1999; $43: 2736-41$

4. Goettsch W., vanPelt W., Nagelkerke N., et al. Journal of Antimicrobial Chemotherapy 2000;46:223-228.

5. Tran J.H., Jacoby G.A. Mechanism of plasmid-mediated quinolone resistance. Proceeding of the National Academy of Science of the United States of America 2002;99:5638-42.

6. Jacoby G.A., Chow N., Waites K.B. Prevalence of plasmid-mediated quinolone reistance. Antimicrobial Agents and Chemoterapy 2003;47:559-62.

7. Wang M., Tran J.H., Jacoby G.A., et al. Plasmid-mediated quinolone resistance in clinical isolates of Escherichia coli from Shanghai, China. Antimicrobial Agents Chemotherapy 2003;47:2242-8.

8. Martínez-Martínez L., Pascual A., Jacoby G.A. Quinolone resistance from a transferable plasmid. Lancet 1998;351:797-9.

9. NCCLS. Methods for dilution antimicrobial susceptibility tests for bacteria that grow aerobically. Approved standard M7-A6, p.1. NCCLS, Wayne, PA, 2003.

10. Pfaller M.A., Sader H.S., Hollis R.J. Chromosomal restriction fragment analysis by pulsed-field gel electrophoresis. In: Isenberg H D (Editor), Clinical Microbiology Procedures Handbook. New York, USA, 1992.

11. Bush K., Jacoby G.A., Medeiros A.A. A funtional classification scheme for b-lactamases and its correlaction with molecular structure. Antimicrobial Agents Chemotherapy 1995;39:121133.

12. Livermore D.M., Williams J.D. $\beta$-lactams: mode of action and mechanisms of bacterial resistance. In: Lorian V (Editor), Antibiotics in Laboratory Medicine. Baltimore, USA, 1996.

13. Bou G., Oliver A., Ojeda M., et al. Molecular characterization of FOX-4, a new AmpC-type plasmid-mediated beta-lactamase from an Escherichia coli strain isolated in Spain. Antimicrobial Agents Chemotherapy 2000;44:2549-53.

14. Lee S.H., Kim J.Y., Lee G.S., et al. Characterization of blaCMY11, an AmpC-type plasmid-mediated beta-lactamase gene in a Korean clinical isolates of Escherichia coli. J Antimicrobiol Chemother 2002;49:269-73.

15. Odeh R., Kelkar S., Hujer A.M., Bonomo R.A. Broad resistance due to plasmid-mediated AmpC beta-lactamase in clinical isolates of Escherichia coli. Clinical Infectious Disease 2002;35:140-5.

16. Eom J.S., Hwang B.Y., Sohn J.W., et al. Clinical and molecular epidemiology of quinolone-reistant Escherichia coli isolated from urinary tract infection. Microbiology Drug Resistance 2002;8:227-34 